\title{
Dual-Polarization Reflectarray in Ku-band Based on Two Layers of Dipole-Arrays for a Transmit-Receive Satellite Antenna with South American Coverage
}

\author{
J. A. Encinar , R. Florencio , M. Arrebola , M. A. Salas , M. Barba , R. R. Boix , G. Toso
}

\begin{abstract}
A 1.1-m reflectarray antenna has been designed, manufactured and tested to fulfil the requirements of a satellite antenna in Ku-band that provides South American coverage in $T x$ and $R x$. The reflectarray cells consist of four dipoles for each polarization in two dielectric layers, which were selected because of their simplicity and high performance. The dipole dimensions are optimized in all the reflectarray cells to accomplish the prescribed radiation patterns, by iteratively calling an analysis routine based on MoM-SD and local periodicity. The measured radiation patterns of the manufactured antenna have been satisfactorily compared with simulations and with a 3-layer reflectarray previously designed for the same mission.
\end{abstract}

Index Terms - Reflectarrays, contoured-beam antennas, dipole arrays, satellite antennas.

\section{INTRODUCTION}

Conventional shaped reflectors are used to provide the very stringent requirements needed for contoured-beam antennas in broadcast and telecommunication satellites, which include, dual-polarization, transmit-receive (Tx-Rx) operation, and high co-polar isolation [1]. Several reflectarray demonstrators have been reported as an alternative to shaped reflectors for contoured-beam satellite antennas [2]-[5]. However, most of them suffer from some limitations in the operation frequencies and do not cover both transmit and receive frequencies in Ku-band.

A 1.2-meter reflectarray has been designed for a Direct Broadcast Satellite (DBS) mission operating in Tx (11.712.2 GHz) and Rx (13.75-14.25 GHz) frequency bands to provide a South American coverage in dual-linear polarization [6]. The results obtained for the manufactured prototype show that a reflectarray can be designed to fulfill the typical requirements of Tx-Rx DBS antennas for the copolar radiation patterns. However, the required level of isolation between orthogonal polarizations in DBS antennas (typically $30 \mathrm{~dB}$ ) is difficult to achieve with the configuration of stacked patches, particularly for a wide contoured beam, as shown in [5]. A new type of reflectarray cell containing two orthogonal sets of parallel dipoles in only two levels of metallization was proposed in [7] to improve the cross-polar properties of reflectarray antennas, to simplify the manufacturing process (respect the reflectarrays made of 3 layers of rectangular patches) and to reduce the antenna cost. The improved performance of the new type of reflectarray was validated by a $40-\mathrm{cm}$ prototype reported in [7].

In this paper, we present the results a 1.1-meter antenna demonstrator designed to fulfill the same requirements as in the case of the 3-layer reflectarray reported in [6], but using the new reflectarray cell proposed in [7] made of two layers with printed dipoles. The results are satisfactorily compared with those obtained for the 3-layer reflectarray.

\section{ANTENNA DESIGN}

\section{A. Antenna Requirements}

The requirements of the "South Pan-American mission (PAN-S)" [6], see Fig. 1, on the Amazonas satellite have been selected to design the new Tx-Rx reflectarray antenna in dual-linear polarization, vertical (V) and horizontal (H). The gain and cross-polar requirements are given in Table I.

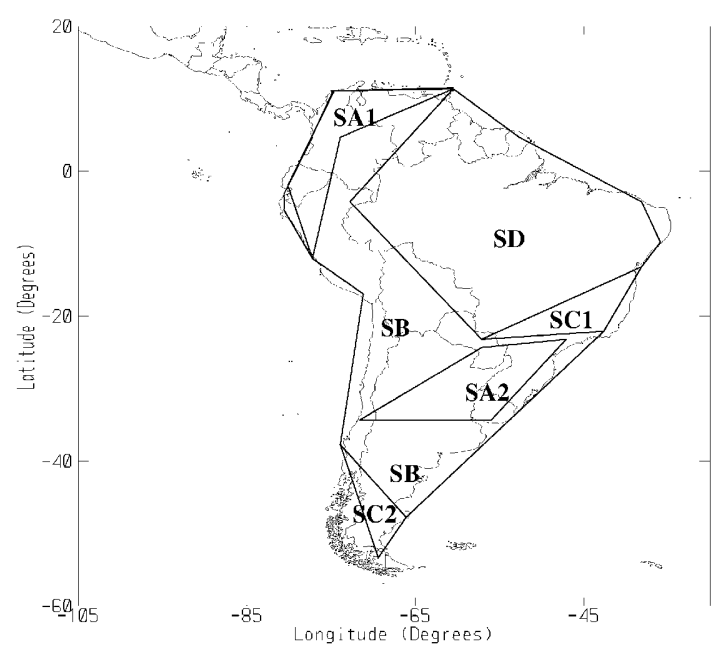

Fig. 1. Coverage of PAN-S mission in Amazonas Satellite. 
TABLE I

GAIN AND CROSS-POL. REQUIREMENTS FOR V-AND H-POLARIZATION

\begin{tabular}{|c|c|c|c|c|}
\hline & \multicolumn{2}{|c|}{ Tx } & \multicolumn{2}{c|}{$\mathrm{Rx}$} \\
\hline Zone & Gain(dB) & XPD $(\mathrm{dB})$ & Gain(dB) & XPI(dB) \\
\hline SA1 & 28.82 & 31.00 & 27.32 & 32.00 \\
\hline SA2 & 28.81 & 31.00 & 27.31 & 28.00 \\
\hline SB & 25.81 & 30.00 & 24.31 & 28.00 \\
\hline SC1 & 22.81 & 29.00 & 22.31 & 28.00 \\
\hline SC2 & 20.66 & 27.00 & 21.28 & 28.00 \\
\hline SD & 19.81 & 27.00 & 18.31 & 25.00 \\
\hline
\end{tabular}

\section{B. Definition of Antenna Breadboard}

The antenna configuration is an elliptical reflectarray panel with axes $1110 \mathrm{~mm} \times 1090 \mathrm{~mm}$. The dimensions of the reflectarray cell have been chosen as $10 \mathrm{~mm} \times 12 \mathrm{~mm}$, and the reflectarray elements are placed in a grid of $110 \mathrm{x} 90$ for $\mathrm{X}$ polarization and $109 \times 89$ for Y-polarization. The antenna contains a total of 7720 elements for X-polarization. The feed for the reflectarray is a circular corrugated horn designed, manufactured and tested by CASA-Espacio (Airbus Defence and Space) [6]. The center of horn aperture is placed at coordinates $(-366,0,1451) \mathrm{mm}$, with respect to the center of the reflectarray panel, see Fig. 2. This antenna configuration provides illumination levels of $-14 \mathrm{~dB}$ in $\mathrm{Tx}$ and $-18 \mathrm{~dB}$ in $\mathrm{Rx}$ at the antenna edge.

The reflectarray cells are based on four dipoles for each polarization in two layers, see Fig. 3. The width of all dipoles is $0.5-\mathrm{mm}$ and the separation between dipoles of the same group is $2.5-\mathrm{mm}$. The lay-up of the reflectarray and the properties of the dielectric layers are shown in Table II. The reflectarray cells are analyzed in a periodic environment using a home-made software based on the Method of Moments in the Spectral Domain (MoM-SD). The phase and amplitude curves have been evaluated for both polarizations in $\mathrm{Tx}(11.7-12.2 \mathrm{GHz})$ and $\mathrm{Rx}(13.75-14.25 \mathrm{GHz})$ frequencies for different angles of incidence. The simulations show a linear phase response in both frequency

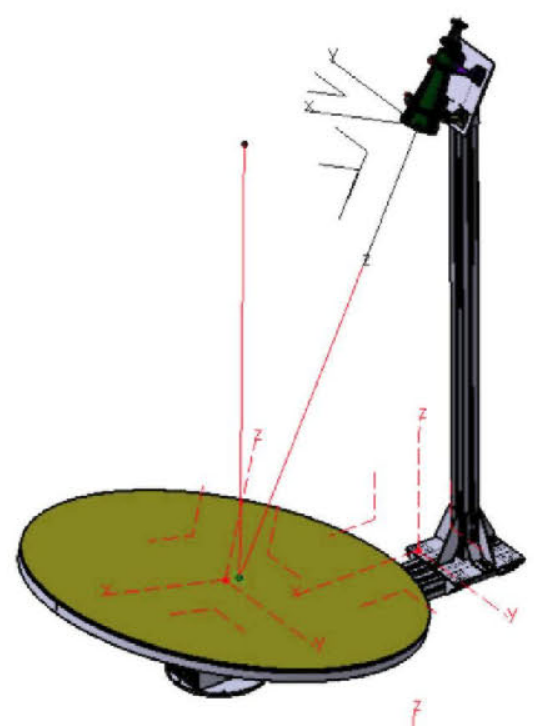

Fig. 2. Drawing of reflectarray antenna demonstrator including the coordinate systems.

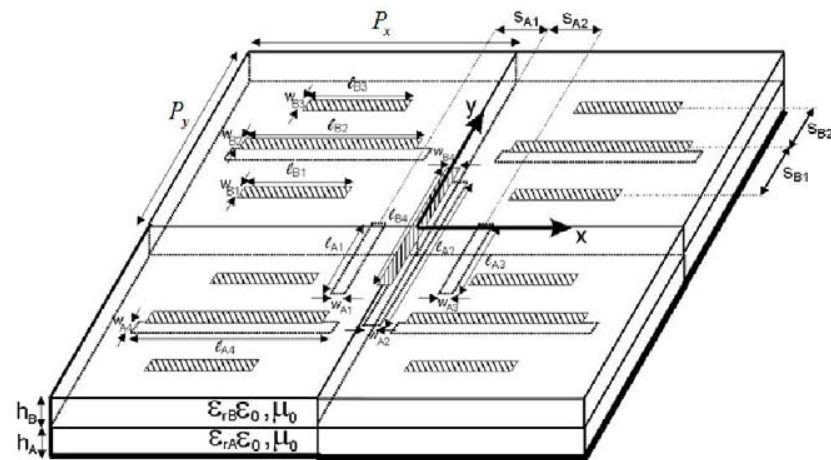

Fig. 3. Reflectarray element based on four parallel dipoles for each polarization in two levels of metallization.

bands, losses lower than $0.2 \mathrm{~dB}$ and cross-polarization below $-15 \mathrm{~dB}$ for most cases of dipole lengths and angles of incidence.

TABLE II. DEFINITION OF LAY-UP

\begin{tabular}{|c|c|c|c|c|}
\hline Layers & Material & $\varepsilon_{r}$ & $\tan \delta$ & $\begin{array}{l}\text { Thickness } \\
\text { (mm) }\end{array}$ \\
\hline Printed dipoles & Copper & - & - & 0.018 \\
\hline B & Diclad $^{\mathrm{TM}} 880$ & 2.17 & 0.0009 & 1.524 \\
\hline Printed dipoles & Copper & - & - & 0.018 \\
\hline Bonding film & CuClad6250 & 2.35 & 0.0025 & 0.038 \\
\hline $\mathrm{A}$ & Diclad $^{\mathrm{TM}} 880$ & 2.55 & 0.0009 & 2.363 \\
\hline Ground plane & Copper & - & - & - \\
\hline
\end{tabular}

\section{Design and Optimization.}

Using the spherical mode expansion for the corrugated horn, the phase distribution required to produce the prescribed coverage has been obtained at different frequencies $(11.7 \mathrm{GHz}, 11.95 \mathrm{GHz}, 12.2 \mathrm{GHz}$ and $14 \mathrm{GHz}$ ) by applying the phase-only pattern synthesis based on the Intersection Approach technique [8]. In a first step, the reflectarray is designed to fulfil the requirements at 11.95 $\mathrm{GHz}$ by using the MoM-SD tool and assuming local periodicity. The resulting dipole dimensions are used as starting point in an optimization run, in which the dipole lengths are optimized to match simultaneously the required phases at central frequency and the phase differences at extreme frequencies in both Tx and Rx bands, as described in [6]. After the first optimization run, the errors in phase and phase differences have been reduced for both polarizations in most part of the reflectarray elements. The optimization process was repeated three times to further optimize the dipole lengths and to improve the antenna performance in both polarizations and frequency bands.

\section{MANUFACTURE OF REFLECTARRAY DEMONSTRATOR}

The printed arrays have been manufactured in three pieces by photo-etching the two faces of the material Diclad $880 \mathrm{~B} 1.524 \mathrm{~mm}$ thick covered by copper $18 \mu \mathrm{m}$ thick. The three panels were correctly aligned and bonded to the grounded dielectric of thickness $2.363 \mathrm{~mm}$. The electrical reflectarray glued to a $20-\mathrm{mm}$ thick aluminum plate and the feed-horn have been correctly positioned by using a supporting structure, as shown in Fig. 2. Fig. 4 shows the 


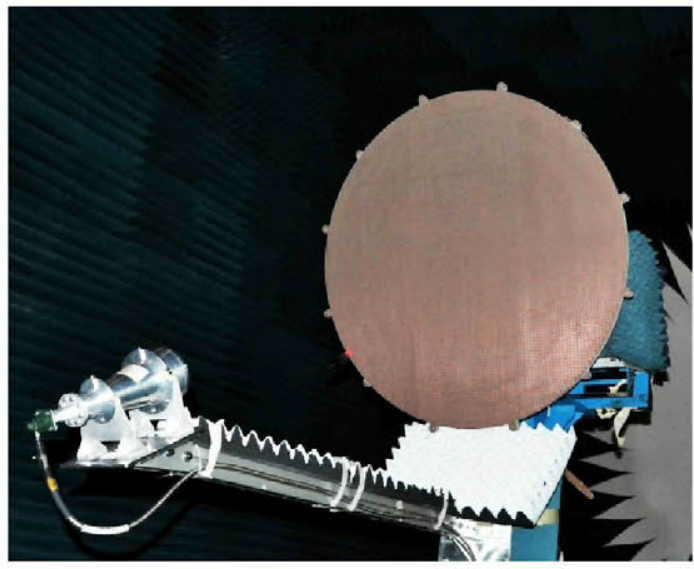

Fig. 4. Reflectarray demonstrator in the UPM anechoic chamber.

final reflectarray demonstrator in anechoic chamber of the Technical University of Madrid (UPM).

\section{COMPARISON OF MEASURED AND SIMULATED RADIATION PATTERNS}

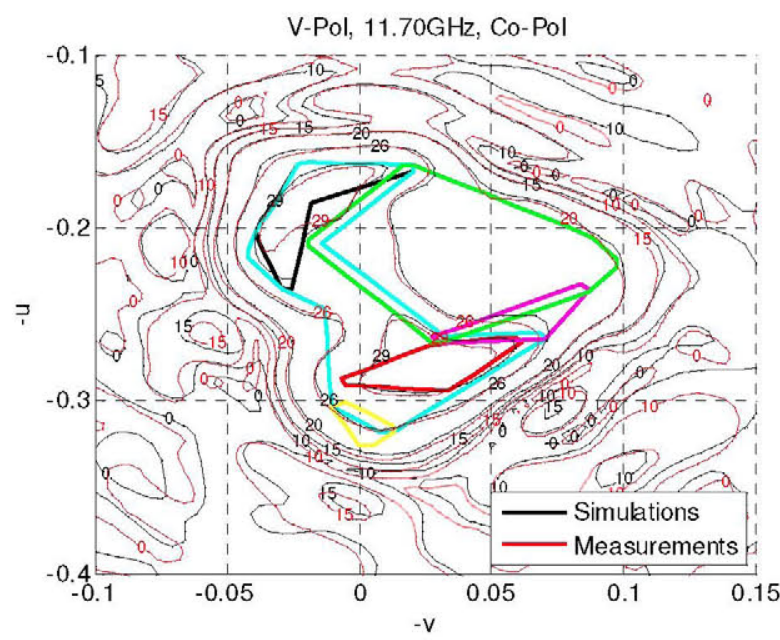

(a)

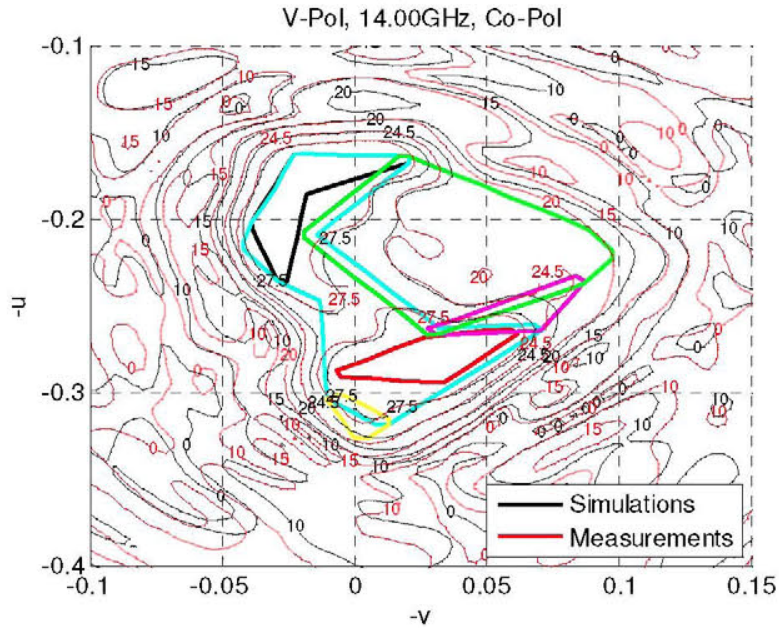

(b)

Fig. 5. Comparison of measured and simulated radiation patterns (Vpolarization) for the reflectarray antenna at $11.7 \mathrm{GHz}$ (a) and $14.0 \mathrm{GHz}$ (b).
The measured radiation patterns are compared with those obtained by the analysis tool developed by UPM based on MoM-SD and local periodicity. Fig. 5 shows the superposition of measured and simulated radiation patterns for V-polarization at $\mathrm{Tx}$ and $\mathrm{Rx}$ frequencies. A good agreement is observed in the co-polar contour lines covering the different zones of the coverage. There are some discrepancies that can be attributed to tolerances in the dipole dimensions, which includes a systematic under etching (oversize of dimensions) of $60 \mu \mathrm{m}$ plus a random error of $+/-20 \mu \mathrm{m}$, and to variation in the dielectric properties of the materials.

\section{COMPARISON WITH 3-LAYER REFLECTARRAY}

The measured co-polar and Cross-Polar Discrimination (XPD) patterns are represented in Figs. 6 and 7 for the demonstrator described here and for the 3-layer reflectarray reported in [6]. To make an easier comparison, the same levels and colors are represented for both demonstrators. The comparison of both results show that the co-polar coverage and the XPD are better accomplished in the new demonstrator than in the 3-layer reflectarray. Similar conclusions are obtained when comparing the patterns for

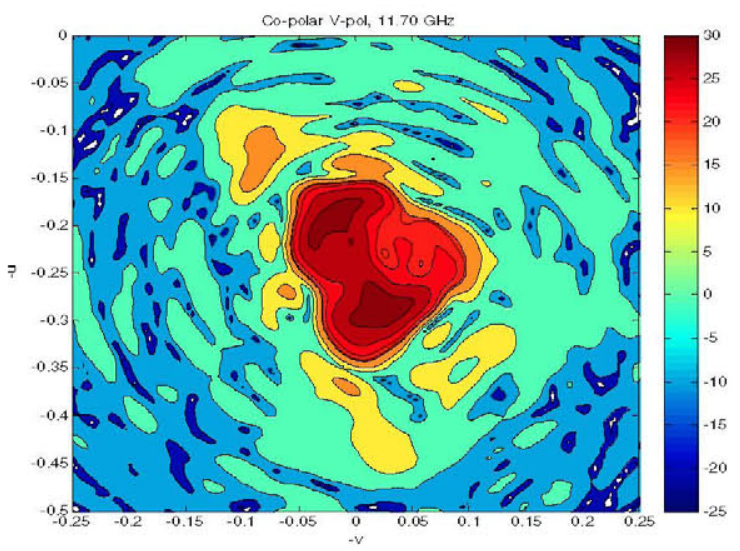

(a)

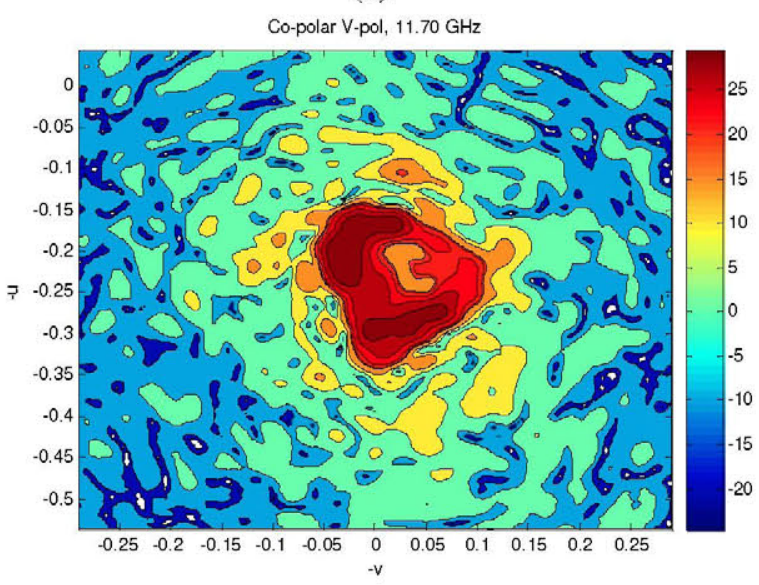

(b)

Fig. 6. Measured radiation patterns at $11.7 \mathrm{GHz}$ for $\mathrm{V}$-polarization for the demonstrator made of dipoles (a) and for the 3-layer reflectarray [6] (b). 


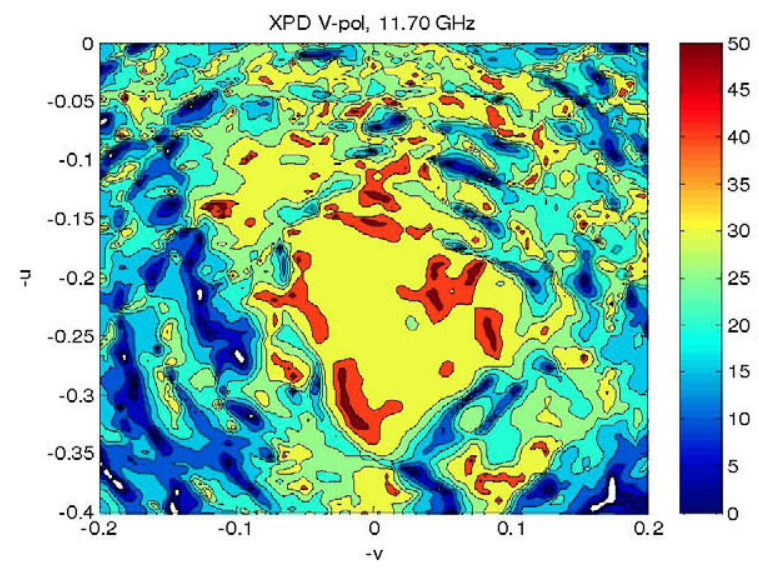

(a)

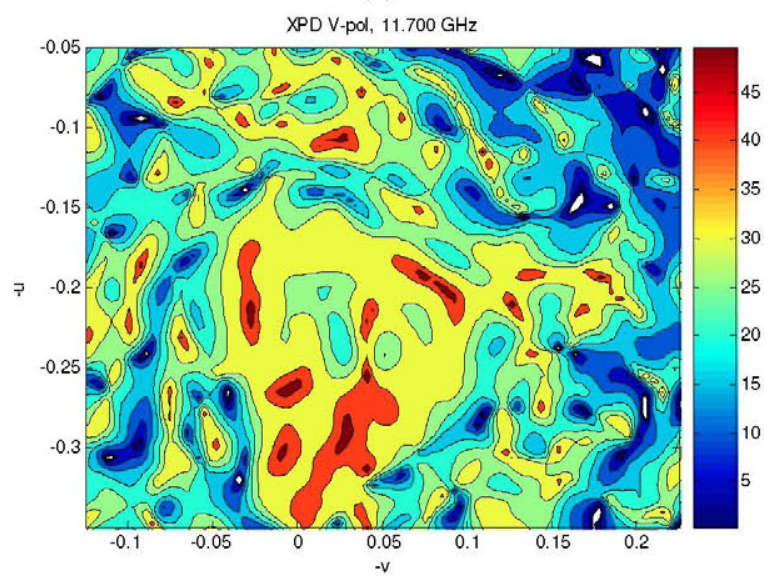

(b)

Fig. 7. Measured XPD at $11.7 \mathrm{GHz}$ for V-polarization for the demonstrator made of dipoles (a) and for the 3-layer reflectarray reported in [6] (b).

other frequencies in both polarizations.

When comparing the reflectarray demonstrator made of dipoles with the 3-layer reflectarray [6], there are several improvements that must be mentioned:

1) The manufacturing complexity has been simplified. Whereas the previous demonstrator required three stacked layers with printed patches, the reflectarray proposed here requires only two layers of printed arrays with dipoles. In the implementation made for the demonstrator, only one bonding film was required. For space technology, two layers of honeycomb and two layers of kapton with printed dipoles are desirable. In any case, the number of layers has been reduced and the electrical performance has been improved.

2) The errors reported in the flatness of the honeycomb panel in the reference antenna have been overcome in the present demonstrator by using a flat rectified aluminum plate $20-\mathrm{mm}$ thick, which ensures flatness better than $0.25 \mathrm{~mm} / \mathrm{m}$.

3) The new reflectarray element proposed here provides lower cross-polarization than the one made of three layers of rectangular patches.

\section{CONCLUSIONS}

A 1.1-m reflectarray demonstrator made of two layers of printed dipoles has been designed, manufactured and tested to fulfil the requirements of a South American coverage in Tx and Rx. The performance of this antenna has been compared with that obtained for a 1.2-m reflectarray made of three layers of stacked patches. The comparison shows that the new reflectarray performs slightly better than the previous one, in spite of the smaller number of layers and smaller antenna size. The results presented here have demonstrated the viability of this technology with very promising capabilities for $\mathrm{Ku}-\mathrm{B}$ and $\mathrm{TX} / \mathrm{RX}$ antennas in communications satellites.

\section{ACKNOWLEDGMENT}

This work has been supported by the Spanish Ministry of Economy and Competitiveness under the project TEC201343345-P, by "Junta de Andalucía" (project P12-TIC-1435) and by the European Space Agency (ESA) under contract 4000117113/16/NL/AF.

\section{REFERENCES}

[1] A. Imbriale, S. Gao, L. Boccia, Space Antenna Handbook", John Wiley \& Sons, 2012 (Ch. 12).

[2] J. Huang and J. A. Encinar, Reflectarray antennas. Piscataway, NJ/New York: IEEE Press/Wiley, 2008 (Ch. 10).

[3] H. Legay, B. Salome, E. Labiole, M.A. Milon, D. Cadoret, R. Gillard, R. Chaharmir, J. Shaker, "Reflectarrays for satellite telecommunication antennas," in Proc. $2^{\text {nd }}$ European Conf. on Antennas and Propagation, EuCAP 2007, Edinburgh, UK, pp. 1-6.

[4] D. M. Pozar, S. D. Targonski, and R. Pokuls, "A shaped-beam microstrip patch reflectarray," IEEE Trans. Antennas Propagat., vol. 47, no. 7, pp. 1167-1173, July 1999.

[5] J. A. Encinar, L. Datashvili, J. A. Zornoza, M. Arrebola, M. SierraCastañer, J. L. Besada, H. Baier, and H. Legay, "Dual-polarization dual-coverage reflectarray for space applications," IEEE Trans. on Antennas and Propag., Vol. 54, no. 10, pp. 2828-2837, Oct. 2006.

[6] J. A. Encinar, M. Arrebola, L. F. de la Fuente, G. Toso, "A Transmit-Receive Reflectarray Antenna for Direct Broadcast Satellite Applications" IEEE Trans. on Antennas and Propagat. Vol. 59, no. 9; pp. 3255-3264, Sept. 2011.

[7] R. Florencio, J. A. Encinar, R. R. Boix, V. Losada and G. Toso, "Reflectarray Antennas for Dual Polarization and Broadband Telecom Satellite Applications," in IEEE Trans. on Antennas and Propag., vol. 63, no. 4, pp. 1234-1246, April 2015.

[8] O. M. Bucci, G. Franceschetti, G. Mazzarella, G. Panariello, "Intersection approach to array pattern synthesis," IEE Proceedings, Vol. 137, Pt. H, No. 6, pp. 349-357, Dec. 1990. 